\title{
Integración industrial de las regiones fronterizas del norte de México a la economía nacional
}

\author{
Alfonso Corona Rentería \\ Universidad Nacional Autónoma de México
}

\begin{abstract}
Resumen
Desde el momento en que se nacionalizó la banca mexicana y se estableció un control de cambios, la frontera norte inició un proceso de reordenación económica, quedando cautivo para el mercado nacional un potencial de cerca de 10 millones de habitantes de la franja fronteriza y cuyo mercado será realidad, sólo si la oferta acude con oportunidad en cantidades y calidades suficientes. Para lograr lo anterior, se sugiere instrumentar políticas de industrialización y comercialización regional; asimismo, plantear objetivos y metas que deberán alcanzarse así como las estrategias y programas que deberán contemplarse. El objetivo de este análisis es descubrir los coeficientes de insumo-producto y la demanda final de las regiones fronterizas respecto de la economía nacional y de Estados Unidos. Igualmente, es posible reproducir los enlaces y los efectos económicos interregionales que puedan lograrse con nuevas inversiones en un sector nacional o internacional.
\end{abstract}

Palabras clave: control de cambios, mercado, nacionalización de la banca, franja fronteriza, comercio transfronterizo.

\begin{abstract}
Since the first time when Mexican banks were nationalized and an exchange control was established, the Northern border of Mexico entered into a process of economic reorganization. In this way, the national market recovered a market of 10 million inhabitants from the border zone, but this potential market will become a reality, only if the offer comes on time on the proper amounts and quality. To achieve this, it is suggested to implement the regional industrialization and marketing policies; at meantime, to propose objectives and goals to be reached, as well as the strategies and programs to be complied. The objective of this analysis is to find out the income-products coefficient as well as the final demand at border zones compared to the domestic economics and of the United States. In same way, it is possible to reproduce the links and inter-regional economical effects that could be achieved through new investments on certain domestic and international sectors.
\end{abstract}

Keywords: exchange control, market, nationalization of banks, border region, transborder trade. 


\title{
INTEGRACION INDUSTRIAL DE LAS REGIONES FRONTERIZAS DEL NORTE DE MEXICO A LA ECONOMIA NACIONAL. *
}

\author{
Dr. Alfonso Corona Rentería \\ Profesor de Carrera \\ Facultad de Economía, \\ UNAM.
}

\section{PREAMBULO}

Cuando en mayo de 1982 los comités organizadores mexicano y norteamericano del Primer Encuentro de Impactos Regionales de las Relaciones Económicas México-Estados Unidos, nos reunimos en cse portento de la naturaleza que es el Gran Cañón del Río Colorado, situado a 427.5 kilómetros al noroeste de esta ciudad ( 253 millas) con el fin de discutir la estructura que se daría al presente segundo encuentro, se acorćó identificar a esta reunión con el nombre de "Desafíos y Oportunidades".

Ha transcurrido un año desde que celebramos aquel seminario preparatorio. La economía mexicana mostraba señales de deterioro, había ocurrido ya la devaluación de febrero de 1982, la primera de las tres que se registraron en ese infausto año. La economía norteamericana se hundía en el receso y el desempleo.

Pero nadie imaginaba que en el curso de un año iban a ocurrir alteraciones tales en las relaciones fronterizas, que darían una nueva y aguda significación actual al lema "Desafíos y Oportunidades".

En efecto, las economías regionales transfronterizas se encuentran ante la coyuntura histórica de recoger el desafío y aprovechar las oportunidades para reordenar su crecimiento y desarrollo conforme a objetivos de reducción de las desigualdades, eslabones económicos y patrones de desarrollo en las cambiantes regiones transfronterizas de México y Estados Unidos.

\section{LAS RELACIONES ECONOMICAS GLOBALES RECIENTES.}

Parece necesario subrayar, de entrada, que el estudio de los impactos regionales de las relaciones económicas entre Estados Unidos y México se plantea en primer lugar, en el contexto global de las interacciones desiguales y jerarquizables de dos países, uno altamente desarrollado, el otro, en vías de desarrollo que presenta un alto grado de dependencia con respecto al primero.

\footnotetext{
- Trabajo presentado en el Segundo Encuentro sobre Impactos Regionales de las Relaciones Económicas México-Estados Unidos.
} 
La disimetría observable en el grado de desarrollo de las regiones situadas de un lado y otro de la frontera es un efecto de la polarización que ejerce el sistema económico del país capitalista más poderoso del mundo frente a su vecino del sur. Los vínculos globales de las economías de cada país se mani fiestan en la dependencia mexicana del mercado norteamericano. Comose sabe, el 69 o/o de las exportaciones mexicanas se dirigen a Estados Unidos y el $63 \%$ de las importaciones procede de aquel país.

Las relaciones de México con el mercado norteamericano se refuerzan mediante las inversiones directas que se orientan en forma creciente hacia $e^{l}$ sector manufacturero y de la alimentación. Un número reducido de empresas norteamericanas son propietarias de la gran mayoría de las inversiones directas extranjeras en México.

La deuda externa mexicana se concentró durante el último decenio en la banca norteamericana por varias razones: el desequilibrio de la balanza de cuenta corriente con ese país, las limitaciones del mercado interno de capitales, el bajo nivel de ahorro y en consecuencia de crédito disponible, el aumento de la tasa de interés bancario en Estados Unidos, las necesidades de financiamiento externo ocasionada por el déficir presupuestal del gobierno federal y las expectativas de crecimiento económico suscitadas por el auge petrolero.

En los años recientes, la economía norteamericana registró los niveles más bajos de desempleo desde la década de 1930 y si bien ahora los indicadores apuntan hacia la recuperación económica, la desocupación no se abatirá substancialmente antes de dos o tres. años. Si a lo anterior se agregan los trastornos graves que sufre la economía mexicana, tenemos un conjunto de relaciones económicas de complejidad creciente con implicaciones políticas y sociales para ambos países que repercuten tanto en la esfera nacional como en el plano internacional.

Durante los años 1979 - 1981 en que se agudizó la recesión mundial, se aceleró el crecimiento de la economía mexicana sobreestimulada por un enorme y deficitario gasto público. Una disminución de los precios del petróleo mexicano a mediados de 1981 junto con el estancamiento de los precios y del volumen de exportaciones mexicanas modificó el curso de la economía mexicana. Para compensar estas pérdidas se contrataron créditos internacionales muy elevados a corto plazo.

Los años de prosperidad en México habían ocasionado un volumen de importaciones extremadamente alto propiciado por la fuerte desgravación arancelaria, la inflación interna aceleraba su velocidad y el desequilibrio de la balanza de pagos alcanzaba niveles sin precedente. La resultante inestabilidad del mercado cambiario se agravó por la especulación monetaria. Se in- 
tensificó el proceso de polarización internacional con la fuga masiva de capitales.

Las enormes inversiones de capitales mexicanos en valores de renta fija y en bienes raíces en ciudades y centros de recreo de Estados Unidos contribuyeron grandemente a la descapitalización de México. Entretanto, ocurrieron las devaluaciones de febrero y agosto de 1982. Ese año disminuyó la afluencia de capitales extranjeros y de turistas a México. El mercado norteamericano se contrajo y continúa reduciéndose para las exportaciones mexicanas. Un ejemplo muy reciente es la exclusión de 57 productos mexicanos del Sistema Generalizado de Preferencias de Estados Unidos.

La contracción del mercado de divisas, el receso de la economía mexicana y las devaluaciones del peso afectaron las importaciones procedentes de Estados Unidos que disminuyeron 60 o/o durante el período comprendido entre el último trimestre de 1981 al último de 1982, en tanto que las estimaciones para 1983 indican una reducción de 10,000 millones de dólares de exportaciones de Estados Unidos a México. Este fenómeno ocasionará el desempleo de unas 231,000 personas de las cuales alrededor de 48,000 residen en la frontera con México.

La segunda devaluación del peso mexicano que marcó el límite inferior de las reservas en divisas, deió como única alternativa la nacionalización de la banca mexicana, el control de cambios y la aparición instantánea del mercado negro en las principales ciudades del país y de la frontera norte. El incremento sin precedentes de la inmigración de trabajadores mexicanos a Estados Unidos es una medida de los efectos sociales de la crisis mexicana. La tercera devaluación decretada en diciembre de 1982 consolidó una nueva realidad para las transacciones fronterizas.

\section{LAS RELACIONES ECONOMICAS FRONTERIZAS.}

El espacio económico y social fronterizo ha sido siempre un reflejo de las intensas y complejas relaciones globales entre ambos países. Compuesto por regiones geoeconómicas diferentes, permeables a los contactos y flujos de personas. bienes, capitales, ideas y presiones políticas, la frontera con sus costos diferenciales permite a los inversionistas estadounidenses aprovechar el menor precio relativo de la mano de obra mexicana para la terminación de manufacturas de empresas transnacionales.

En el pasado reciente, la ausencia de una oferta nacional de bienes intermedios y de productos terminados y la nula integración interindustrial de bs estados fronterizos así como la sobrevaluación del peso mexicano favorecida por el régimen de zona libre permitió un gigantesco comercio de importación de bienes consumo que restaba, al mismo tiempo, las posibilidades de una industrialización regional integrada a la economía nacional. 
La crisis mexicana puso de relieve en forma espectacular un aspecto de la fuerte interdependencia asimétrica de las ciudades gemelas fronterizas que antes se soslayaba. Esto es la dependencia de los establecimientos comerciales norteamericanos respecto de la clientela mexicana que, al suspenderse las operaciones cambiarias con los inabordables nuevos tipos de cambio, no pudo adquirir ya los dólares necesarios para hacer sus compras cotidianas de bienes duraderos y semiduraderos en las tiendas del lado norteamericano.

Por ejemplo, las exportaciones originadas en San Diego se redujeron 16 o/o desde el primer trimestre de 1981 al último de 1982. Esta cifra es semejante en la mayoría de las ciudades estadounidenses fronterizas con México. El efecto ha sido desvastador sobre la economía de las ciudades fronterizas norteamericanas a lo largo de tres mil kilómetros de frontera.

En el lado mexicano de la frontera, un 15 o/o de los comercios cerró por falta de mercancía importada. La planta industrial trabaja con menor intensidad por la carencia de insumos de importación. El abastecimiento de mercancías es insuficiente para satisfacer una demanda que ha aumentado $300 \mathrm{o} \%$.

Ahora nos encontramos con dos economías vecinas de desarrollo desigual que han sufrido considerablemente como resultado de las crisis de ambos países. Cabe preguntarse si este desorden podría presentarse en dos economías transfronterizas menos dependientes del comercio al menudeo, más vinculadas entre sí por flujos de insumo-productos interindustrial y más integradas a las economías de sus respectivos países.

Desde que se nacionalizó la banca y se estableció el control de cambios. la frontera norte inició un proceso de reordenación económica; el dólar no es ya la moneda imperante en cualquier transacción y el peso ha tomado el lugar que le corresponde.

Queda cautivo para el mercado nacional un potencial de 10 millones de habitantes de la franja fronteriza que antes compraba sus alimentos del lado americano. Otro número semejante de mexicanos residentes en el sur de Estados Unidos constituye igualmente un mercado potencial para las exportaciones mexicanas. Ambos mercados serán una realidad sólo si la oferta nacional acude con oportunidad en cantidades y calidades suficientes. Para ello se requiere instrumentar políticas de industrialización y comercialización regional basados en la complementación de los eslabones intersectoriales de bienes intermedios (insumos) y productos terminados entre los estados fronterizos y con respecto al resto de la economía. Los eslabones intersectoriales e interregionales de insumo producto deben reforzarse con enlaces interurbanos de Este a Oeste y de Norte a Sur. 
Si no se actúa con rapidez, la elevada inflación doméstica podrá devolver en el término de uno o dos años su ventaja ancestral a los precios de las manufacturas estaounidenses sin que hayamos podido rescatar para México los mercados de nuestra frontera. Los aumentos recientes del precio de la gasolina mexicana que ahora, con su calidad inferior, es más cara que la americana, han hecho resurgir el espectro de la elevada propensión a importar bienes de consumo de aquel país.

Si se actúa con imaginación, sentido de equidad y racionalidad, el beneficio que México puede derivar de su integración industrial-regional mediante la creación de empleos y la elevación de los niveles de vida en los estados fronterizos, permitirá construir una red de intercambios de bienes intermedios y productos terminados que complemente procesos productivos interregionales orientados hacia los mercados domésticos e internacionales. Es probable que en contrapartida se requiera mejorar la integración regional de las economías del sur de Estados Unidos. De esa manera, el comercio fronterizo ocupará un lugar importante en la economía de esas regiones pero habrá dejado de ser la actividad económica predominante y por lo tanto será mucho menos vulnerable a los cambios cíclicos o estructurales típicos del sistema capitalista.

\section{LAS RELACIONES ECONOMICAS ENTRE EL NORTE Y EL CENTRO DE MEXICO.}

Desde el punto de vista regional, la integración interindustrial de los estados fronterizos se justifica debido a que la contribución de esas entidades a la economía nacional es relativamente pequeña considerando el tamaño de su territorio, su potencial de desarrollo y escasa población.

En efecto, los estados norteños con más de 44 o/ de la superficie del territorio nacional sólo contaban en 1980 con el 16.1 o/ de la población y apenas alcanzaron ese año el 20.2 o o del producto interno bruto en su conjunto. Con excepción de Chihuahua, el resto de los estados fronterizos alcanzaron un producto interno bruto per cápita superior al promedio nacional.

La participación de la región dentro de la actividad económica nacional, en el año de 1980 se caracterizó por una intervención en la composición del producto interno bruto de $22.9 \mathrm{o} / \mathrm{o}$ en el sector agropecuario, $21.2 \mathrm{o} / \mathrm{o}$ en el de la distribución, $21.2 \mathrm{o} / \mathrm{o}$ en el sector electricidad, $20.1 \mathrm{o} / \mathrm{o}$ en la industria manufacturera, 18.3 o/o en la minería y 17.8 o/o en la construcción.

La densidad de población de habitantes por kilómetro cuadrado de los estados fronterizos es inferior al promedio nacional, con excepción del estado de Nuevo León que es ligeramente superior; de las cincuenta principales localidades urbanas de la república, 16 corresponden a los estados del Norte. 
Los problemas que afrontan las economías de los estados norteños son entre otras, un marcado desequilibrio entre la oferta y la demanda de bienes y servicios generadas en la región, una deficiente integración económica de la zona; la dispersión geográfica de los principales centros urbanos y la falta de comunicación interestatal y con el resto de la república. Esta situación ha limitado las posibilidades de desarrollo de buen número de actividades que requieren de un mercado ampliado, así como de aquellas que cuentan con posibilidades de exportación; a lo anterior se agrega el problema del flujo de personas que llegan a la frontera con el objeto de trabajar en el vecino país.

El espacio territorial y su potencial de recursos humanos naturales y la posibilidad de generar en la zona un comercio con una mayor participación de bienes de origen nacional, justifica el estudio para el desarrollo de los estados del Norte en su conjunto.

Si bien las industrias maquiladoras han logrado su objetivo de generar empleo, sus fundamentos legales limitan las posibilidades de tenerlas en cuenta como una fuente estable de ocupación y su producción de bienes actualmente no influye en el desarrollo y la integración industrial del país.

Las relaciones económicas entre los estados del Norte y los del centro. se componen principalmente de la exportación hacia el centro de productos de la siderurgia, maquinaria y equipo, sector agropecuario y pesca. A su vez. el centro exporta un gran número de bienes que sin embargo resultan insuficientes para el consumo de las regiones fronterizas.

En consecuencia, se estima urgente poner en marcha una política de integración industrial e interregional que contribuya a resolver los probelmas enunciados. Esta política debe contemplar los mercados norteamericanos para el aprovisionamiento de insumos y para la exportación de productos nacionales.

La formulación de la política de referencia tendría los siguientes:

\section{OBJETIVOS Y METAS}

General: Con prioridad nacional. Disminuir la dependencia de la econo. mía de los estados fronterizos respecto de Estados Unidos. Incrementar la contribución de las economías de las regiones de la frontera a la economía y al desarrollo nacional.

General. Contribuir a la descentralización industrial y demográfica de la zona conurbada del centro del país, favoreciendo el desarrollo de ciudades medianas así como la disminución del subempleo, y la creación de empleos y la distribución regional del ingreso. 
Particular. Formular un modelo de política de integración interregional, intersectorial e internacional de las industrias de los estados del norte con las del centro y sur de México.

Un resultado cuantitativo que se espera obtener de la investigación, es proponer un conjunto de actividades productivas apoyadas por recursos locales e insumos interregionales y/o procedentes de Estados Unidos, señalando el ámbito del mercado que puede ser nacional o internacional.

El resultado cualitativo es el conjunto de disposiciones de política de industrialización integrada regionalmente Norte-Sur, así como recomendaciones de políticas de incentivos a la inversión en los sectores propuestos de actividad en las localidades seleccionadas. El modelo de política de integración tendrá congruencia con los diferentes planos de desarrollo industrial.

Particular. Selección de un número de localidades en los estados fronterizos que conforme a una estrategia de ubicación geográfica, tamaño, demografía, dotación de recursos naturales, económicos, humanos, infraestructuras y equipamiento urbanos, sean adecuados para la localización de inversiones industriales y agropecuarios articuladas intersectorial e interregionalmente.

Particular. Diseñar un conjunto de políticas complementarias aplicables en diferentes esferas económicas y sociales; instrumentos fiscales y monetarios, tipo de cambio, comercio exterior, redes de abastecimiento comercial, distribución del gasto familiar, capacitación para el trabajo, etc. que refuerce la política interindustrial y que sea congruente con los objetivos de integración de la economía nacional.

Los objetivos anteriores, puede verse, cubren un campo muy vasto. Más allá de los aspectos puramente económicos, incluyen dimensiones sociales, políticas, del medio ambiente, etc. Sin embargo, el objetivo privilegiado es el incremento de la producción regional del norte de México. La realización de este objetivo económico deberá contribuir a la realización de objetivos conjuntos como la reducción de bolsas regionales de subempleo, una mejor distribución del ingreso entre regiones, etc. A los instrumentos de política económica como las inversiones públicas directas en las regiones, los subsidios y controles, etc. se agrega un conjunto de instrumentos no económicos adaptados a las necesidades y niveles de desarrollo regional, como los de política de energéticos, infraestructuras, vivienda, educación, protección del medio ambiente. La eficacia relativa de los instrumentos es función de su pertinencia para movilizar los factores de producción y localización que requieren específicamente ciertos sectores de producción. 


\section{ESTRATEGIAS DE POLITICA DE INTEGRACION INDUSTRIAL RE- GIONAL.}

La política de desarrollo industrial regional debe descansar sobre una estrategia con dos dimensiones; una especial, la otra sectorial. La primera $\$$ relaciona a la localización, tamaño y número de ciudades seleccionadas para la asignación de inversiones. La segunda se refiere a la adecuación de los sectores y de las ramas industriales que se proponen para ciudades prioritarias. En este respecto se utilizan los instrumentos de análisis de factores de localización.

El número de ciudades por desarrollar forma parte de la estrategia de industrialización, ya sea descentralización concentrada en un pequeño número de centros industriales o bien, descentralización dispersa en numerosas ciudades. Aquí se trata de un problema de elección estratégica. Hay autores que consideran que el mejor tamaño urbano para el crecimiento industrial en México, es una población de 500,000 habitantes. Según este criterio, sólo las ciudades de Tijuana y Mexicali en Baja California y Ciudad Juárez, Chihuahua, situadas en la frontera norte, reunirían esa condición. Estas ciudades registran un ritmo de crecimiento extremadamente alto, de donde se derivan muchos problemas de hacinamiento, están muy polarizadas por el lado norteamericano y es preferible regular y mantener estable su crecimiento, favoreciendo así a otras ciudades medianas del interior de esas entidades.

Es muy importante recordar aue la integración de los estados del Norte con el conjunto del país, depende de la posibilidad de crear nuevos eslabones técnicos sectoriales así como de la existencia de una red muy activa de localidades y carreteras que reduzcan los tiempos de los viajes al centro de México. En este caso debemos encontrar la mejor combinación de tamaños y localización geográfica que permita a una localidad llenar los dos criterios: economías externas y de la urbanización capaces de atraer las inversiones industriales. El otro criterio es el potencial de recursos locales que permitirán el despegue industrial urbano susceptible de integración intersectorial e interregional con el resto del país.

La selección de industrias a partir del análisis de vínculos interindustriales es particularmente apropiada para la identificación de un pequeño número de industrias clave que una vez establecidas en las regiones de referencia, deben inducir un proceso de crecimiento rápido e innovador. Deben estimular el progreso tecnológico y ser industrias exportadoras en la región. Estas industrias requieren intensidad de capital de inversión, mejores vínculos interregionales y menores niveles de ocupación.

El otro enfoque, mencionado antes, preconiza, por lo contrario, una política de diversificación industrial que hace a la región en vías de desarrollo menos vulnerable a las fluctuaciones cíclicas y por lo tanto favorece una 
mayor estabilidad del empleo y del ingreso regional. Estas industrias pueden utilizar mayor intensidad de trabajo que las anteriores, mantener mayores vínculos intrarregionales y menores de carácter interregional.

El grado de aislamiento de una región es un factor decisivo en estos casos. A mayor aislamiento y mayores distancias que recorrer para llegar a un centro regional importante, mayores serán las tendencias hacia la utilización intensiva de la fuerza de trabajo y una integración hacia adentro, es decir, intrarregional. Por otro lado, las regiones escasamente desarrolladas pero abiertas y con buenos vínculos con las regiones centrales tienen capacidad para absorber fuerza de trabajo, mantener fuertes eslabones intrarregionales y propiciar un proceso de industrialización con un mejor equilibrio entre la utilización intensiva de capital y de mano de obra.

Las anteriores reflexiones son útiles para adoptar criterios de estrategia en cuanto a la concentración o dispersión de las plantas industriales así como a la intensidad relativa de capitales o mano de obra en los proyectos de inversión regional. No obstante la utilización del análisis de flujos de insumoproductos parece favorecer de entrada los proyectos bien integrados interregionalmente y que requieren altos volúmenes de capital y poca intensidad relativa de mano de obra. a.

Es deseable que el modelo de integración industrial intersectorial, interregional e internacional propuesto, se construya en torno a los conceptos de umbral de crecimiento y de perfiles regionales que permitan, en el marco de la teoría de la localizacion, descubrir los factores determinantes de actividades industriales específicas así como cuantificar la influencia de estos factores sobre el crecimiento sectorial de las regiones en estudio a fin de situarlas con relación a un umbral crítico de crecimiento.

El umbral de crecimiento puede referirse a regiones en expansión o estancadas. Puede escogerse una variable representativa del crecimiento de regiones, por ejemplo, la tasa de incremento del empleo o de la producción, para que sirva como indicador sintético de las características regionales. A csa variable se le asigna un valor que funge como escala de medición. Por ejemplo, si Ina región obtiene un coeficiente o tasa de crecimiento del empleo o de la producción inferior a esa escala podrá considerarse como perteneciente al żupo de regiones estancadas; si, por lo contrario, obtiene una puntuación superior a ese valor crítico, pertenecerá al grupo de regiones en crecimiento. El concepto de umbral servirá también como parámetro cuando se trate de establecer un diagnóstico del estado de las regiones atrasadas. Este diagnósico permitirá por una parte, identificar los elementos específicos sobre los que habrá que actuar y por la otra, el tipo de política de desarrollo que permita a esas regiones a franquear el umbral y crear las condiciones para iniciar d proceso de crecimiento. 
El segundo elemento es la noción de indicador de atracción regional. $\mathrm{El}$ indicador se compone de un subconjunto de factores de localización pertinentes para el sector considerado, en el sentido de que si una región tiene suficiente dotación de estos factores, será atractiva para el establecimiento de actividades nuevas y para la ampliación de actividades existentes del sector en cuestión. El conjunto de todos los factores de localización potenciales constituye el "perfil regional", representado por un coeficiente que puede leerse como "propensiones a atraer actividades sectoriales". El valor de estas propensiones indica la importancia del factor de localización correspondiente, al determinarse la atracción que ejerce la región sobre el sector. Su contenido puede organizarse en un cierto número de perfiles, como el de atracción propiamente dicho, compuesto esencialmente de elementos de oferta y demanda intermedia y final, el perfil clásico que corresponde a la disponibilidad de factores de producción que no son otra cosa que recursos naturales, económicos y humanos localizados en una región o centro de población específico, el perfil sociocultural o político, que se refiere a las instituciones y a las políticas regionales, el medio ambiente, etc. Todos estos elementos pueden estar presentes en las regiones con intensidades variables y su pertinencia para la localización de actividades sectoriales específicas variará de un sector a otro. La política de desarrollo regional se propone modificar el perfil (diagnóstico) regional con el fin de crear las condiciones favorables al crecimiento cuando aquellas no existan.

El modelo considera explícitamente las interdependencias regionales. Las regiones de nuestro estudio no son sistemas cerrados: por lo contrario, tanto los mercados de insumos como los de productos en la mayoría de los sectores industriales modernos, poseen una dispersión espacial que se extiende mucho más allá que las fronteras de la región escogida. Igualmente, las interdependencias tecnológicas tienen una dimensión espacial interregional a partir de las localizacionẹs de actividades industriales en una región dada; esto es así, no por razón de la atracción propia de la región sino porque ésta es contigua a un importante mercado (por ejemplo, el mercado estadounidense) para los productos del sector o como relaciones espaciales entre diferentes regiones que ofrecen un mercado de productos, acceso a materias primas, la tercera, un conjunto de servicios necesarios al funcionamiento del sector, etc.

\section{PROGRAMAS}

I. Análisis de flujos interregionales, intersectoriales, internacionales que señale:

A. Qué insumos se producen en los estados fronterizos susceptibles de incorporarse a los procesos productivos en otras regiones mexicanas. Se determinarán las áreas de mercado del producto final. 
B. Qué insumos originados en alguna de las regiones mexicanas pueden incorporarse a los procesos de producción de las regiones del norte de México. El mercado puede ser nacional o internacional.

C. Qué insumos de origen norteamericano se requieren para integrar las regiones de los estados fronterizos, horizontal y verticalmente con eslabones fuertes respecto al resto del país.

D. Demanda final de regiones fronterizas respecto de la economía nacional y de Estados Unidos.

La matriz de flujos sobre el origen y destino interregional de los insumos permitirá identificar lineas de producción con ventajas comparativas para el mercado nacional, y para la exportación, oferta y demanda nacional y externa y localización regional. Se evaluará la contribución de estas actividades al logro de objetivos de descentralización de la actividad económica, creación de empleos, integración económica interregional, disminución del déficit de la balanza de comercio con Estados Unidos, desarrollo de ciudades medianas y otros.

Si la información sobre flujos lo permite, se elaborarán matrices de intercambio para cada estado del Norte poniendo de relieve los coeficientes regionales de oferta de algunos sectores (los sectores locales están compuestos por la producción local). Un modelo paradigmático puede proponer que el 60 o 70 por ciento de la demanda regional de ciertos bienes esté cubierta por la producción local. El resto de la demanda regional puede ser abastecido por otras regiones, ya sean mexicanas o americanas. Para reunir las condiciones del paradigma de integración regional es necesario una matriz de tres sectores: el de regiones fronterizas, las otras regiones mexicanas, especialmente Monterrey, Guadalajara y la región metropolitana del Distrito Federal, Toluca y Puebla, y el sector norteamericano1

El objetivo del análisis anterior es descubrir los coeficientes de insumoproducto y la demanda final de las regiones fronterizas respecto de la economía nacional y de Estados Unidos. Igualmente, es posible reproducir los enlaces y los efectos económicos interregionales que pueden lograrse con nuevas inversiones en un sector nacional o internacional.

\footnotetext{
Tuna razón importante para separar los componentes regionales de una matriz de flujos intersectoriales macionales es que en cada país la distribución de actividades económicas es muy desigual entre las diferentes regiones. Por este motivo, un cambio en la actividad tendrá impactos desiguales en los niveles del ingreso y del empleo. Además, un modelo regional destaca los efectos generales para las diferentes técnicas de producción, hábitos del consumidor y modalidades del comercio entre regiones.
} 
Factores de localización:

El modelo de integración interregional se apoya en una estrategia de des centralización de establecimientos industriales distribuidos en un número de localidades de los estados fronterizos que por su ubicación geográfica y recursos presenten viabilidad para un desarrollo sostenido de los espacios regionales subpoblados que separan el norte del centro de México. Con este fin se incorpora el análisis de los factores de localización de la actividad económica regional.

II.- Inventario de recursos.

Evaluación de las características geográficas, recursos naturales, económicos y humanos de las regiones urbanas seleccionadas para determinar su potencial de recursos para el desarrollo.

III.- Desarrollo urbano.

1) Armazón urbano y estructura de funciones y servicios de las ciudades en estudio

2) Región polarizada de cada ciudad

3) Infraestructura y equipamientos urbanos

4) Redes interurbanas

5) Sistemas y jerarquías de ciudades interfronterizas en el marco regional IV.- Perfiles de inversión.

Cartera de proyectos de preinversión: la información contendrá un número manejable de proyectos viables que pueden localizarse en los estados de la frontera norte.

1. Estudio de mercado: demanda, oferta, precios, comercialización, el producto.

2. Estudio técnico: tamaño proceso productivo y localización; obras físicas, organización.

3. Estudio financiero: recursos financieros para la inversión; análisis y proyecciones financieras; financiamiento.

4. Evaluación económica: relaciones entre el proyecto y el conjunto de la economía; efecto previsto del proyecto. 
V.- Modelo de política

Es un trabajo de síntesis y de armonización de programas, que equivale a un modelo de política de industrialización con las características descritas en las páginas precedentes. 